\title{
In-Situ TOF-SIMS and SFM Measurements Providing True 3D Chemical Characterization of Inorganic and Organic Nanostructures
}

\author{
Ewald Niehuis $^{1 *}$, Rudolf Moellers ${ }^{1}$, Felix Kollmer ${ }^{1}$, Henrik Arlinghaus ${ }^{1}$, Laetita Bernard ${ }^{2}$, Hans Josef \\ Hug $^{2}$, Sasa Vranjkovic ${ }^{2}$, Raphaelle Dianoux ${ }^{3}$, Adi Scheidemann ${ }^{3}$ \\ ${ }^{1}$ ION-TOF GmbH, Muenster, Germany \\ 2 EMPA, Duebendorf, Switzerland \\ ${ }^{3}$ Nanoscan AG, Duebendorf, Switzerland
}

Information on the chemical composition, physical properties and the three dimensional structure of materials and devices at the nanometer scale is of major importance in nanoscience and nanotechnology. Time-of-Flight Secondary Ion Mass Spectrometry (TOF-SIMS) is known to be an extremely sensitive surface imaging technique which provides elemental as well as comprehensive molecular information on all types of solid surfaces. Depth profiling of multilayers with high depth resolution as well as threedimensional analysis is performed in the so-called dual beam mode. In this mode the pulsed analysis beam is combined with a low energy sputter ion beam for the removal of material providing chemical 3D information of the sample. However, the topography of the initial sample surface as well as the subsequent evolution of the topography due to different erosion rates of the compounds cannot be identified by the technique and lead to distortions of the detected depth distribution. In order to get a true image of the 3D volume, the time scale of the TOF-SIMS 3D depth profiles needs to be converted into a depth scale. Scanning Force Microscopy (SFM) provides the required complementary information on the surface topography with a resolution on the nanometer level. Beyond that SFM can provide valuable information about the physical properties of the sample if the cantilever is operated in the different dynamic operation modes.

We have combined the techniques ToF-SIMS and SFM in one UHV analysis chamber. This instrument was conjointly developed within the framework of a FP7 research project (www.3dnanochemiscope.eu). One of the key components of the new instrument is a piezo driven XYZRT-stage which moves the sample between the TOF-SIMS and the SFM analysis site with sub- $\mu \mathrm{m}$ precision and high speed. This allows analyzing exactly the same surface area with both techniques.

The TOF-SIMS analysis is performed using a new bismuth liquid metal cluster ion gun that can achieve a beam size for $\mathrm{Bi}_{3}$ cluster primary ions down to $20 \mathrm{~nm}$ [1]. These heavy projectiles exhibit very high secondary ion yields in particular for organic materials. For the sputtering of inorganic materials the instrument is equipped with an oxygen and cesium beam. At low sputter energies of a few hundred $\mathrm{eV}$ a depth resolution of about $1 \mathrm{~nm}$ is achieved for inorganic thin films. Although this beam energy is rather low, the sputtering of organic materials would lead to strong radiation damage and a rapid loss of the molecular ion signals. This is avoided by the use of large gas clusters with energies per atom in the range from $1-10 \mathrm{eV}$ [2] allowing high quality molecular depth profiles of organic multilayers with a depth resolution of $5 \mathrm{~nm} \mathrm{[3]} \mathrm{as} \mathrm{well} \mathrm{as} \mathrm{3D} \mathrm{analysis} \mathrm{of} \mathrm{organic} \mathrm{nanostructures} \mathrm{[4].}$

The SFM unit with a beam deflection design is mounted on a 3-axis high precision flexure stage scanner. This linearized scanner with a very small out-of-plane motion yields very accurate information on the surface topography and has a scan range of $40 \times 40 \times 6 \mathrm{~m}^{3}$. The SFM is also used to measure the depth of the sputter craters, which typically have a size of several hundred $\mu \mathrm{m}$. A special long distance surface profiler mode was developed to measure the correct shape and depth of the sputter crater. Several cantilevers with different properties for the various SFM modes of operation are stored in the 
UHV chamber allowing a fast exchange of the cantilever. The SFM can be operated in contact mode as well as in a variety of dynamic modes including KPFM and MFM.

In this paper we will present first results illustrating the strength of combined in-situ TOF-SIMS / SFM measurements and the potential for a wide range of applications including sputter induced effects on the surface morphology of inorganic as well as organic solid surfaces. The procedure for 3D analysis by combined SFM and ToF-SIMS measurements is explained using a thin film of a polystyrene (PS) polymethracrylate (PMMA) blend on a $\mathrm{Si}$ wafer as a model system. The surface topography in the analysis area of $30 \times 30 \mu \mathrm{m}^{2}$ was analyzed by SFM followed by ToF-SIMS dual beam 3D analysis in the same area using $\mathrm{Bi}_{3}$ analysis combined with large Ar cluster sputtering. Finally, the resulting topography was measured again by the SFM. In addition, the total depth of the sputter crater with a size of $300 \times 300 \mu \mathrm{m}^{2}$ was determined in the profiler mode of the SFM. The 3D ToF-SIMS data were stored in a raw data file that consists of the $\left(\mathrm{x}, \mathrm{y}, \mathrm{t}_{\text {sputter }}, \mathrm{m}\right)$ coordinates for each detected secondary ion. The retrospective data analysis of the 2D and 3D SIMS data makes use of multivariate statistical analysis methods such as PCA and MCR. Finally the 3D data of the main components are combined with the SFM topography data and crater depth to get the correct 3D representation of the analytical volume $(\mathrm{x}, \mathrm{y}, \mathrm{z}, \mathrm{m})$. Figure 1 shows the SFM image at the surface and the overlay with the SIMS data for the two main components PS and PMMA. We will also show an example for the detection of Ag nanoparticles in macrophages from a mouse. Living cells on a $\mathrm{Si}$ wafer were exposed for different times to $\mathrm{Ag}$ nanoparticles with a diameter of $200 \mathrm{~nm}$. For the analysis the cell culture was cleaned with PBS and fixated with glutaraldehyde. SFM and ToF-SIMS results will be shown for single cells sputtered by large Ar clusters.
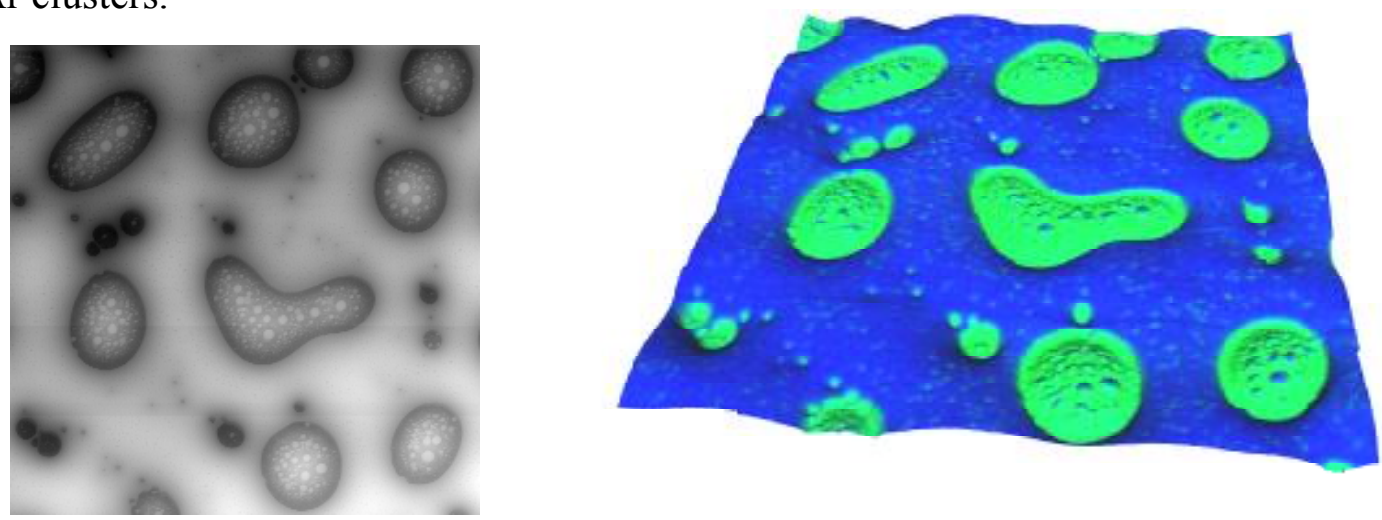

Figure 1. a) SFM image of a PS/PMMA polymer blend (left) b) overlay of the SIMS distributions measured for the two materials (green PMMA, blue PS)

\section{References}

[1] F. Kollmer, W. Paul, M. Krehl and E,. Niehuis, Surf. Interface Anal. 45, 312 (2013)

[2] S. Ninomiya, K. Ichiki, H. Yamada, Y. Nakata, T. Seki, T. Aoki and J. Matsuo,Rapid Commun. Mass Spectrom. 23, 3264 (2009)

[3] A.G. Shard, R. Havelund, M.P. Seah, S.J. Spencer, I.S. Gilmore, N. Winograd, D. Mao,T. Miyayama, E. Niehuis, D. Rading, R. Moellers, Anal. Chem. 84(18) (2012), 7865

[4] E. Niehuis, R. Moellers, D. Rading, H.-G. Cramer, R. Kersting, Surf. Interface Anal.45, (2013) 158

Acknowledgement

The authors acknowledge funding from the European Commission (FP7, Grant number 200613). 